\title{
Histopathological evaluation of the ocular-irritation potential of shampoos, make-up removers and cleansing foams in the bovine corneal opacity and permeability assay
}

\author{
Masatoshi Furukawa ${ }^{1}$, Takashi Sakakibara ${ }^{1},{\text { Kouta } \text { Itoh }^{1} \text {, Kohtaro Kawamura }}^{1}$, Satoshi Sasaki ${ }^{*}$, \\ and Masao Matsuura ${ }^{1}$
}

${ }^{1}$ Safety Research Institute for Chemical Compounds Co., Ltd., 363-24 Shin-ei, Kiyota-ku, Sapporo, Hokkaido 004-0839, Japan

\begin{abstract}
The bovine corneal opacity and permeability (BCOP) assay is an alternative method to the in vivo Draize eye test in rabbits for evaluating eye irritation in vitro. Here, we compared the numerical results of the BCOP assay with the corresponding histopathology for three different corneas for each test substance, including commercially available shampoos, make-up removers and cleansing foams that contained surfactants and other ingredients. The histopathological score was defined based on the severity of lesions in the corneal epithelium. The histopathological findings and scores of the three sections for each test substance were comparable. The in vitro irritancy score (IVIS) generally corresponds to the corneal irritant potential of the test substances assigned on the basis of the histopathological findings in this study. In the present study, we characterized the histopathology of the corneal epithelium and stroma and especially showed that the corneal epithelial injury caused by test substances might be important in assessment of test substances that are mild eye irritants (category 2B) as classified by the United Nations (UN) Globally Harmonized System of Classification and Labelling of Chemicals (GHS), as corneal lesions suggestive of classification into category 2B were localized on the border between the corneal epithelium and stroma, which contained cell elements related to assessment of prognosis of an in vivo eye injury. Histopathological assessment might be useful in predicting in vivo ocular irritation, particularly for test substances with an IVIS $>3.1$ but $\leq 25$ that are classified as mild irritants (category 2B) according to the UN GHS. (DOI: 10.1293/tox.2015-0022; J Toxicol Pathol 2015; 28: 243-248)
\end{abstract}

Key words: bovine corneal opacity and permeability (BCOP) assay, IVIS, cosmetic products, eye irritation, histopathological scoring, corneal epithelium

\section{Introduction}

The standard bovine corneal opacity and permeability (BCOP) assay (without histology) is generally accepted as a valid alternative in vitro method to the Draize eye irritation test ${ }^{1}$. The BCOP assay has been shown to be suitable for identifying moderate, severe and very severe eye irritants classified as categories $2 \mathrm{~A}$ and 1 according to the Globally Harmonized System of Classification and Labelling of Chemicals (GHS). However, this assay has not yet been proven to discriminate accurately between moderately irritating (United Nations [UN] GHS category 2A/2B) and mildly irritating compounds (UN GHS category $2 \mathrm{~B})^{2}$. In the

Received: 27 April 2015, Accepted: 25 June 2015

Published online in J-STAGE: 31 July 2015

*Corresponding author: S Sasaki

(e-mail: sasaki-satoshi@ka-anken.co.jp)

(C2015 The Japanese Society of Toxicologic Pathology

This is an open-access article distributed under the terms of the Creative Commons Attribution Non-Commercial No Derivatives (by-ncnd) License $<$ http://creativecommons.org/licenses/by-nc-nd/3.0/> .
BCOP assay, opacity is determined by the amount of light transmission through the cornea, and permeability is determined by the quantity of sodium fluorescein dye that passes through the corneal cell layers. Both measurements are summed to provide the in vitro irritancy score (IVIS) to predict the in vivo ocular irritation potential of a test substance. The mean IVIS is then graded according the OECD Guideline $437,2013^{2}$, as follows: no category (IVIS $\leq 3$ ), no prediction can be made (IVIS $>3$ to 55 ), category 1 (IVIS $>55$ ). In 2014, the guidelines of the Pharmaceutical and Food Safety Bureau (PFSB) Guidance (2014) ${ }^{3}$ designated irritants with an IVIS $\leq 3$ as "non-irritant." The Interagency Coordinating Committee on the Validation of Alternative Methods (ICCVAM) recommended the following in vitro score ranges for the BCOP assay: a substance with a score ranging from 0 to 3.0 is classified as "not labeled," one with a score ranging from 3.1 to 25 is classified as "mild irritant" suggestive of UN GHS category $2 \mathrm{~B}$, one with a score ranging from 25.1 to 55 is classified as a "moderate irritant" suggestive of UN GHS category 2A and one with a score of more than 55.1 is classified as a "severe irritant" suggestive of UN category $1^{4}$. In histopathological evaluation in an ex vivo rabbit cor- 
neal assay, representative full thickness cross sections of cornea were prepared; and the epithelium, stroma and endothelium were examined; and the depth of corneal injury was evaluated to determine the extent of corneal injury as follows: slight irritants damage the superficial epithelium, mild irritants penetrate further to damage the stroma, and severe irritants penetrate through the cornea and damage the endothelium $^{5,6}$, as determined by the recommended EPA catego$\mathrm{ry}^{7}$. The depth of injury is a mechanistic correlate of the ocular irritation response based on ocular tissues comprising both the epithelium and the stroma. However, assessment of corneal epithelial injuries resulting from substances classified into UN GHS category $2 \mathrm{~B}$ has been insufficient to date, as the lesions are localized to the border zone between the corneal epithelium and the corneal stroma, which contain wing cells, basal cells and the basement membrane, which are directly related to the reversibility of a corneal injury.

In this study, we investigated the histopathology of eyes after application of cosmetic products that resulted in an IVIS of 0 to 3.0 or 3.1 to 25 , which were classified into the "not labeled" or "mild irritant" category, respectively", and showed more practical evidence corroborating the corneal irritation of test substances for the prediction of in vivo irritancy.

\section{Materials and Methods}

This study was conducted in accordance with OECD Test Guideline $437^{2}$.

Shampoos were diluted to $10 \%$ with purified water for testing or not diluted. Make-up removers and cleansing foams were not diluted for testing. Distilled water was used as a solvent and as a negative control. Undiluted N,Ndimethylformamide was used as a positive control. The $\mathrm{pH}$ values of the test substances were determined using $\mathrm{pH}$ paper (Whatman Cat No. 2600-100A; GE Healthcare, Buckinghamshire, UK).

Eyes were obtained from 12- to 60-month-old cows at an abattoir (Hokkaido Chikusan Corporation) as a byproduct from fleshly slaughtered animals and immediately transferred to Safety Research Institute for Chemical Compounds Co., Ltd. in cold Hanks' balanced solution. Upon arrival at the laboratory, the corneas with defects (including cuts, scratches, small opaque areas and neovascularization) were discarded. Three separate eyes were prepared for each test substance.

Bovine corneas with 2 to $3 \mathrm{~mm}$ around the sclera remaining to assist in subsequent handling were excised and maintained in Hanks' balanced solution. Corneas were mounted in specially designed corneal holders, and the two compartments of the holder (anterior and posterior chambers) were filled with Eagle's minimum essential medium (phenol red-free) and 1\% fetal bovine serum. Mounted corneas were incubated at $32 \pm 1^{\circ} \mathrm{C}$ for $1 \mathrm{~h}$ to allow equilibration.

An initial opacity reading was conducted for all cor- neas using a calibrated opacitometer (BASF-OP3.0, BASF; DURATEC Analysentechnik GmbH, Hockenheim, Germany). The medium was removed from the anterior chamber of the holder, and then $750 \mu \mathrm{l}$ of each test article or control substance was applied to the epithelial surface for $10 \mathrm{~min}$. After exposure, the test article or control substance was removed, the corneas were thoroughly rinsed at least 3 times with Eagle's medium containing phenol red, the chambers were refilled with fresh Eagle's medium (phenol red-free), and the corneas were incubated at $32 \pm 1^{\circ} \mathrm{C}$ for $2 \mathrm{~h}$. The second opacity reading was taken using the opacitometer after incubation.

After this post-exposure opacity measurement, the medium in both chambers was aspirated, and the posterior chamber was refilled with fresh medium, while the anterior chamber receiving $1 \mathrm{~mL}$ sodium fluorescein solution. The Chambers were then incubated vertically (so that the corneal surface keeps a bar level with the ground) for $90 \mathrm{~min}$. Following this incubation, the medium in the posterior chamber was removed and placed into a pre-labeled glass tube. The optical density of each medium at $490 \mathrm{~nm}\left(\mathrm{OD}_{490}\right)$ was determined using a spectrophotometer, and the following formula was used to determine the permeability $\left(\mathrm{OD}_{490}\right)$ :

Permeability value $\mathrm{OD}_{490}=\left(\right.$ each value $\mathrm{OD}_{490}-$ blank value $\mathrm{OD}_{490}$ ) - negative control value $\mathrm{OD}_{490}$

The net change in opacity (corrected opacity value) for each treated cornea was obtained by subtracting the initial (baseline) opacity reading from the final opacity reading. The mean opacity for each test substance and that for the negative control were used to calculate the IVIS using the following formula:

IVIS $=$ corrected mean opacity value $+(15 \times$ mean permeability value $\mathrm{OD}_{490}$ )

Means and standard deviations were calculated using Sharp Scientific Calculator (Model EL-501E).

Immediately after the BCOP assay, corneas were fixed in $10 \%$ neutral buffered formalin solution and embedded in paraffin, and $6-\mu \mathrm{m}$-thick sections were stained with hematoxylin and eosin. Histopathological changes of the corneal epithelium, stroma, and endothelium were investigated, and the lesions of the corneal epithelium were graded as follows: 0 , unremarkable; 1 . slight; 2 , mild; 3 , moderate; and 4 . severe. Grades were assigned according to the location of the lesion, as follows: squamous cells (slight), between the squamous and upper wing cells (mild), from squamous to underneath wing cells (moderate) and over basal cells (severe). The irritation potential of test substances in the corneal stroma was evaluated based on the extent of loose collagen bundles and swelling of keratocytes, as follows: collagen matrix damage extending no further than the upper third of the corneal stroma (mild irritant), extending no further than two-thirds of the way through the corneal stroma (moderate irritant) and extending into the lower third of the corneal stroma and/or causing damage to the endothelial cells (severe irritant) according to the histopathological decision criteria of the Academy of Managed Care Pharmacy Back- 
Table 1. The Means and Standard Deviations of Opacity, Permeability $\left(\mathrm{OD}_{490}\right)$ and IVIS and $\mathrm{pH}$ Values of the Test Substances

\begin{tabular}{llcrrr}
\hline Treatment & $\begin{array}{c}\text { Physical properties } \\
\text { of test solution }\end{array}$ & $\mathrm{pH}$ & $\begin{array}{c}\text { Opacity } \\
\text { mean } \pm \mathrm{SD}\end{array}$ & $\begin{array}{c}\text { Permeability } \\
\text { mean } \pm \mathrm{SD}\end{array}$ & $\begin{array}{c}\text { IVIS } \\
\text { mean } \pm \mathrm{SD}\end{array}$ \\
\hline Distilled water & Liquid & 6 & $0.0 \pm 1.0$ & $0.003 \pm 0.002$ & $0.0 \pm 0.985$ \\
$100 \%$ N-N-dimethylformamide & Liquid & 6 & $79.0 \pm 6.351$ & $1.019 \pm 0.242$ & $94.3 \pm 9.582$ \\
$10 \%$ Shampoo A & Non-viscid fluid & 6 & $0.6 \pm 0.577$ & $0.003 \pm 0.002$ & $0.6 \pm 0.608$ \\
$100 \%$ Shampoo A & Semi-viscid fluid & 6 & $1.6 \pm 1.155$ & $0.408 \pm 0.124$ & $7.7 \pm 2.940$ \\
$10 \%$ Shampoo B & Non-viscid fluid & 6 & $0.3 \pm 1.0$ & $0.03 \pm 0.011$ & $0.8 \pm 1.106$ \\
$100 \%$ Shampoo B & Semi-viscid fluid & 6 & $1.0 \pm 0.577$ & $0.214 \pm 0.098$ & $4.2 \pm 2.050$ \\
$10 \%$ Shampoo C & Non-viscid fluid & 7 & $0.3 \pm 1.0$ & $0.134 \pm 0.058$ & $2.3 \pm 1.193$ \\
$100 \%$ Shampoo C & Semi-viscid fluid & 6 & $0.6 \pm 1.155$ & $0.308 \pm 0.198$ & $5.2 \pm 3.710$ \\
$10 \%$ Shampoo D & Non-viscid fluid & 6 & $0.6 \pm 0.577$ & $0.185 \pm 0.065$ & $3.4 \pm 1.510$ \\
$100 \%$ Shampoo D & Semi-viscid fluid & 6 & $2.0 \pm 0.577$ & $0.659 \pm 0.216$ & $11.9 \pm 3.769$ \\
$10 \%$ Shampoo E & Non-viscid fluid & 7 & $1.3 \pm 0.0$ & $0.222 \pm 0.049$ & $4.6 \pm 0.737$ \\
$100 \%$ Shampoo E & Semi-viscid fluid & 6 & $3.0 \pm 1.528$ & $0.853 \pm 0.305$ & $15.8 \pm 6.105$ \\
$100 \%$ Make-up remover A (oil type) & Non-viscid fluid & 6 & $0.4 \pm 1.528$ & $0.003 \pm 0.007$ & $0.4 \pm 1.419$ \\
$100 \%$ Make-up remover B (liquid type) & Semi-viscid fluid & 6 & $0.7 \pm 0.0$ & $0.009 \pm 0.014$ & $0.8 \pm 0.231$ \\
$100 \%$ Make-up remover C (gel type) & Semi-viscid fluid & 7 & $3.4 \pm 0.577$ & $0.269 \pm 0.061$ & $7.4 \pm 1.474$ \\
$100 \%$ Cleansing foam A & Viscid fluid & 9 & $3.4 \pm 2.082$ & $0.326 \pm 0.161$ & $8.3 \pm 4.179$ \\
$100 \%$ Cleansing foam B & Semi-viscid fluid & 9 & $13.0 \pm 1.528$ & $0.552 \pm 0.075$ & $21.3 \pm 2.621$ \\
\hline
\end{tabular}

SD: standard deviation. Our current historical control data (IVIS) for distilled water range from -0.3 to 0.3 , and those for N-Ndimethylformamide range from 66.6 to 103.3 .

ground Review Document (AMCP BRD) ${ }^{4}$.

\section{Results}

Table 1 shows the means and standard deviations for opacity, permeability $\left(\mathrm{OD}_{490}\right)$ and IVIS and $\mathrm{pH}$ values of the test substances.

Although the standard deviations for the IVIS showed high variability in some test substances, the histopathological findings were comparable amongst the three slides for the test substance.

1. Negative control (distilled water) (IVIS, 0.0; opacity: 0.0 ; permeability, $0.003 ; \mathrm{pH}, 6$ ): The outermost squamous cells exhibited a slightly crude cell matrix, causing desquamation of superficial cells during the life cycle of the corneal epithelium. The histopathological score was graded as 0 (unremarkable) (Fig. 1A).

2. Positive control (100\% N-N-dimethylformamide) (IVIS, 94.3; opacity, 79.0; permeability, 1.019; pH, 6): Epithelial thickness and rarefaction of epithelial cells with various vesicles and pyknotic nuclei were prominent in the squamous cell and wing cell layers. The basal cells and cells transitioning to wing cells exhibited a spindle form in the appreciable extracellular space. Loose collagen bundles and swelling of keratocytes were observed in the upper half of the corneal stroma (arrow). Corneal endothelial cells remained intact. The histopathological score was graded as 4 (severe) (Fig. 1B).

3. "No category" (IVIS $\leq 3)$ : The histopathological score was low (slight), as lesions were limited to the squamous cell layer.

a) $100 \%$ make-up remover A, oil-type (IVIS, 0.4; opacity, 0.4; permeability, 0.003; $\mathrm{pH}, 6$ ): Squamous cells with a crude matrix and swollen nuclei were observed in the outermost squamous cells. No pathological changes were ob- served in the other elements in the corneal epithelium and corneal stroma. Corneal endothelial cells remained intact. The histopathological score was graded as 1 (slight) (Fig. 1C).

b) $10 \%$ shampoo C (IVIS, 2.3; opacity, 0.3; permeability, 0.134; pH, 7): The squamous cells of the corneal epithelium exhibited continuous hydropic swelling with fusiform and swollen nuclei. The internal squamous cell layer exhibited no marked pathological changes, and the stratified structure of the corneal epithelium was maintained. No pathological changes were observed in wing cells, basal cells, the basement membrane, Bowman's layer and the corneal stroma. Corneal endothelial cells remained intact. The histopathological score was graded as 1 (slight) (Fig. 1D).

4. "No prediction can be made" (IVIS $>3$ to 7): The histopathological score was still relatively low (mild), and lesions were limited to the squamous cell and upper wing cell layers.

a) $10 \%$ shampoo E (IVIS, 4.6; opacity, 1.3; permeability, $0.222 ; \mathrm{pH}, 7$ ): Squamous cells became round or oval in shape and cloudy in appearance. Marked hydropic changes were observed in rows of upper wing cells. Wing cells in direct contact with the lesion increased in cell density and exhibited nuclear condensation, particularly in the region of transition of basal cells to wing cells. No pathological changes were observed in basal cells, the basement membrane or Bowman's layer. No marked change was observed in the corneal stroma. Corneal endothelial cells remained intact. The histopathological score was graded as 2 (mild) (Fig. 1E).

5. "No prediction can be made" (IVIS $>7$ to $\leq 55)$ : The histopathological score was moderate, with lesions limited to the squamous cell layer and the upper and lower wing cell layers, or severe, with lesions extending over basal cells.

a) $100 \%$ make-up remover C, gel-type (IVIS, 7.4; opac- 


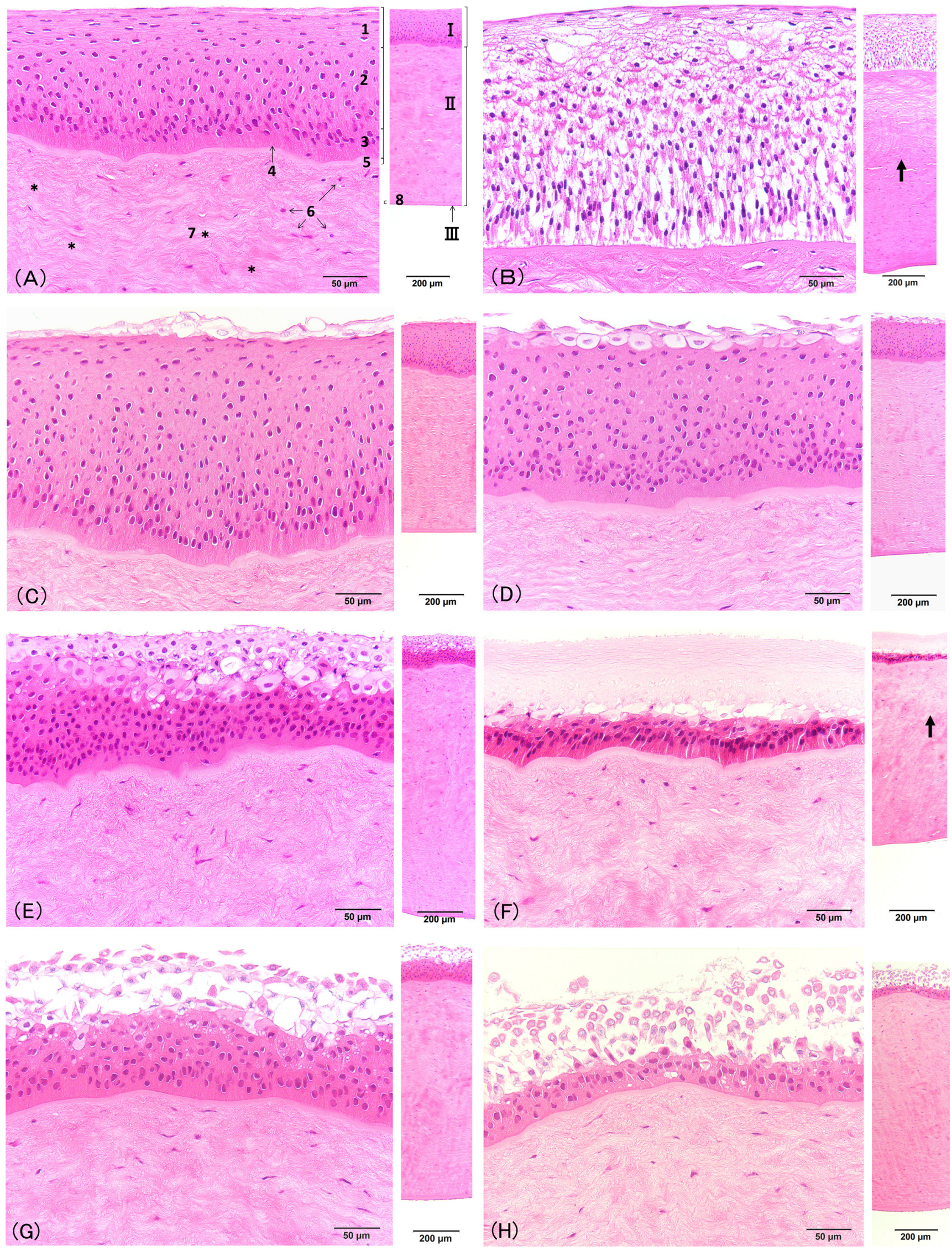

Fig. 1. 
ity, 3.4; permeability, 0.269; $\mathrm{pH}, 7$ ): Epithelial cell damage characterized by hypochromic staining and loss of the nucleus or by observation of disintegration of the nucleus from the squamous to deep wing cell layers. These findings appeared to be the typical microscopic characteristics of ghost cells. Basal cells remained intact. Swelling of keratocytes and loose collagen bundles were observed in the upper third of the corneal stroma (arrow). Corneal endothelial cells remained intact. The histopathological score was graded as 3 (moderate) (Fig. 1F).

b) $100 \%$ cleansing foam A (IVIS, 8.3; opacity, 3.4; permeability, 0.326; $\mathrm{pH}$, 9): Epithelial cell loss and loss of intercellular adhesion in the remaining cells were observed. Wing cells in the region of transition of basal cells to wing cells remained in the layers containing small vacuoles. No marked changes were observed in the corneal stroma. Corneal endothelial cells remained intact. The histopathological score was graded as 3 (moderate) (Fig. $1 \mathrm{G}$ ).

c) $100 \%$ cleansing foam B (IVIS, 21.3; opacity, 13.0; permeability, 0.552; $\mathrm{pH}, 9)$ : Marked corneal epithelial cell loss was observed. The remaining cells were not formed in linear layers. Basal cells remained in layers containing vacuoles. No remarkable changes were observed in the corneal stroma. Corneal endothelial cells remained intact. The histopathological score was graded as 4 (severe) (Fig. 1H).

These histopathological findings of the corneal epithelium and severity generally corresponded to the in vitro score range. A case of $100 \%$ make-up remover C, gel-type, was graded as 3 (moderate) on the basis of the corneal epithelial damage, although the stromal damage extended no further than the upper third in the corneal stroma (Fig. 1F).

\section{Discussion}

In this study, we investigated the histopathological characteristics observed in the BCOP assay using cosmetic products. We showed that histopathological classification of lesions in the corneal epithelium might be useful in predicting the more actual corroboration of ocular irritation of test substance in vitro classification of mild irritant.

Eyes can be exposed to cosmetic products such as mascaras and eye creams and diluted products such as shampoos that are not intended to enter the eye when undiluted. The evaluation of potential eye irritation caused by cosmetic products is essential to ensure that a product is safe for both intended uses and accidental exposures to the eyes. Histopathological tissue analysis of the corneas in the BCOP assay might be useful in evaluating the objective potential for corneal irritation caused by test substances.

The BCOP assay consists of two primary end points to indirectly measure the damage (irritation potential) caused by topically applied test substances: corneal opacity and corneal permeability to fluorescein. Opacity is affected by the direct precipitation or denaturation of proteins in the corneal epithelium, which can result in cloudy swelling on histopathological analysis. Further, the combination of stromal swelling, (i.e., swelling of keratocytes and loose collagen bundles, with increased separation of the collagen fibrils) enables evaluation of the severe irritation potential of test substances.

Epithelial cells possess intercellular junctions, such as tight junctions and adherent junctions, for epithelial adhesion and barrier functions. Tight junctions are the most apical structure of the cornea and obliterate intercellular space to provide a permeability barrier. Disruption of tight and adherent junctions between cells therefore increases epithelial permeability ${ }^{8}$. Degeneration and necrosis of the corneal epithelium or loss of the epithelial layers might markedly influence the irritation potential of products coming into contact with the eye.

The corneal epithelium rapidly responds to injury. Corneal epithelial cells originate from basal cells, which consist of a single layer of columnar cells. The surface of the cornea consists of the stratified squamous epithelium, which requires continuous renewal, and the corneal epithelial cell, which form layers and originate from basal cells. Basal cells migrate from the apical surface and differentiate into wing cells and then squamous cells. The surface of the stratified squamous epithelium is shed continuously as debris. The corneal epithelium is completely replaced approximately every two weeks in rats ${ }^{9}$ and approximately every seven days in adult humans ${ }^{10}$. Corneal defects resurface due to an active repair process. Cell-to-cell and cell-to-matrix interactions play important roles in maintenance of the stratified structure of the corneal epithelium. In response to epithelial damage, remaining epithelial cells migrate to repair the defective area. The condition of the basement membrane is an important factor during wound healing in the corneal epithelium. When the cornea is mechanically scraped, the original basement membrane might survive intact and be re-

Fig. 1. Histopathology of the bovine cornea. HE stain. (A) Negative control (distilled water). IVIS, 0.0; score, unremarkable. I: Corneal epithelium. II: Corneal stroma. III: Corneal endothelium. 1 Squamous cell layer, 2 Wing cell layer, 3 Basal cell layer, 4 Basal lamina, 5 Bowman's lamina, 6 Keratocytes, 7 Lamellar collagen $(*), 8$ Descemet's membrane. The cornea consists of five layers from the anterior to posterior, as follows: corneal epithelium, Bowman's layer, corneal stroma consisting of collagen bundles and keratocytes (fibrocytes), Descemet's membrane and corneal endothelium. The bovine corneal epithelium is composed of 15 to 17 rows with various types of stratified epithelium. From the surface layer to the basement membrane, there are approximately 4 to 5 rows of flattened "squamous" cells (superficial cells), 10 to 13 rows of wing cells and a single row of columnar basal cells in contact with the basement membrane. (B) Positive control (100\% N-N-dimethylformamide). IVIS, 94.3; score, severe. (C) 100\% make-up remover A, oil-type. IVIS, 0.4; score, slight. (D) 10\% shampoo C. IVIS, 2.3; score, slight. (E) 10\% shampoo E. IVIS, 4.6; score, mild. (F) 100\% make-up remover C, gel-type. IVIS, 7.4; score, moderate. (G) 100\% cleansing foam A. IVIS, 8.3; score, moderate. (H) 100\% cleansing foam B. IVIS, 21.3; score, severe. 
used by new epithelial cells. Wing cells participate in reepithelialization during wound healing and can be found at the leading edge in contact with the basement membrane ${ }^{11}$.

In conclusion, we showed that the IVIS in the BCOP assay generally corresponds to the corneal irritant potential of cosmetic products and that histopathological assessment of the corneal epithelium might provide a more reliable evaluation of the eye irritation potential of test substances with an in vitro BCOP score of 0 to 3 (classified as "not labeled") or 3.1 to 25 (classified as "mild irritant"). Histopathological assessment of a corneal epithelial injury would provide a method for examining the prognosis of an in vivo eye injury.

Disclosure of Potential Conflicts of Interest: The authors declare no conflict of interest.

\section{References}

1. Sina JF, Gautheron P, Casterton P, Evans MG, Harbell JW, Curren RD, Earl LK, and Bruner L. Report from the bovine corneal opacity and permeability technical workshop. In Vitro Mol Toxicol. 11: 315-351. 1998.

2. OECD Guidelines for the testing of chemicals. Bovine Corneal Opacity and Permeability Test Method for Identifying i) Chemicals Inducing Serious Eye Damage and ii) Chemicals Not Requiring Classification for Eye Irritation or Serious Eye. 26 July 2013.

3. Guidance of Bovine Corneal Opacity and Permeability Test
(BCOP) for safety evaluation of cosmetics and quasi drugs. PFSB Notification No. 0204. February 4 2014; (in Japanese).

4. US environmental protection agency office of pesticide programs. Non-animal testing approach to EPA labeling for eye irritation. May 112009.

5. Jester JV, Li L, Molai A, and Maurer JK. Extent of initial corneal injury as a basis for alternative eye irritation tests. Toxicol In Vitro. 15: 115-130. 2001. [Medline] [CrossRef]

6. Maurer JK, Parker RD, and Jester JV. Extent of initial corneal injury as the mechanistic basis for ocular irritation: key findings and recommendations for the development of alternative assays. Regul Toxicol Pharmacol. 36: 106-117. 2002. [Medline] [CrossRef]

7. Background Review Document of an In Vitro Approach for EPA Toxicity Labeling of Anti-Microbial Cleaning Products. EPA Toxicity Labeling Background Review Document. EPA BRD-Final Report.

8. Niessen CM. Tight junctions/adherens junctions: basic structure and function. J Invest Dermatol. 127: 2525-2532. 2007. [Medline] [CrossRef]

9. Cenedella RJ, and Fleschner CR. Kinetics of corneal epithelium turnover in vivo. Studies of lovastatin. Invest Ophthalmol Vis Sci. 31: 1957-1962. 1990. [Medline]

10. Hanna C, Bicknell DS, and O'Brien JE. Cell turnover in the adult human eye. Arch Ophthalmol. 65: 695-698. 1961. [Medline] [CrossRef]

11. Danjo Y, and Gipson IK. Specific transduction of the leading edge cells of migrating epithelia demonstrates that they are replaced during healing. Exp Eye Res. 74: 199-204. 2002. [Medline] [CrossRef] 